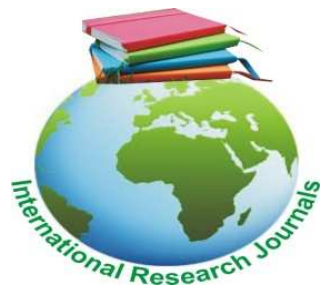

Educational Research (ISSN: 2141-5161) Vol. 9(1) pp. 016-020, January, 2018

Available online@ http://www.interesjournals.org/ER

DOI: http:/dx.doi.org/10.14303/er.2018.017

Copyright (@ 2018 International Research Journals

Review

\title{
Sexuality: assessment, challenges and way forward in nursing profession
}

\author{
${ }^{*}$ Adewole Abimbola D and Ojewale Margaret $O$ \\ Department of Nursing, College of Medcine, University of Ibadan, Ibadan, Oyo State \\ *Corresponding Author's Email:dokkybammy@yahoo.com, +2348034797939
}

\begin{abstract}
Sexual health is a major health need and concern for patients recovering from major diseases and disorders. Adequate understanding of patient's sexuality can contribute to the period of time a patient spends on admission. Therefore, it is very important that health professionals especially nurses understands individual patient's needs of sexuality to be able to give holistic care. Sexuality is therefore, a central component of all human functions that need to be fulfilled. The World Health Organization defined sexual health as "a state of physical, emotional, mental and social well-being in relation to sexuality; it is not merely the absence of disease, dysfunction or infirmity" (WHO, 2012). In order words, sexuality is a part of who you are as a person. It is how you feel about your body, whether you feel masculine or feminine or somewhere in between, the way you dress, how you move, how you speak, the way you act and feel about other people, who you are attracted to and fall in love with, and so much more. According to WHO, 2012, the way people experience and express sexuality is different for each individual and can be influenced by biologic, psychological, social, cultural, and religious factors. When a patient is ill and on admission, the sexuality of the patient is altered especially, with patients that the illness affects their reproductive organs and damage or removal of a part of their body. However, nurses who are part of the health team and relate with patients more on the ward must be well knowledgeable and sensitive to determining the level of alteration in sexuality of the patient or clients they are treating. However, not all the nurses have adequate knowledge of sexuality and the nurses that have the understanding of sexuality do not put it to practice due to one reason or the other. The word sexuality have therefore been reviewed in order to broaden the knowledge of nurses on sexuality, assessment of sexuality, importance of assessing, challenges of assessment of sexuality and way forward in nursing profession.
\end{abstract}

Keywords: Sexuality, Assessment, Challenges, Way forward and Nursing Profession.

\section{INTRODUCTION}

World Health Organization's (WHO's) has defined sexuality as "A central aspect of being human throughout life and encompasses sex, gender identities and roles, sexual orientation, eroticism, and social well-being that relates to one's sexuality, (WHO, 2012). WHO defined sexual health as: "Physical, emotional, and social wellbeing that relates to one's sexuality." Each patient defines their sexuality and sexual health differently and uniquely according to gender, age, personal attitudes, and religious and cultural beliefs (National Cancer Institute, 2012). However, whether you are on your own or sexually involved with someone else, you are still a sexual person. Everyone has their own way of expressing their sexuality to others and we each also have our own way of feeling or experiencing it for ourselves. The individual sexual activity is defined as "any mutually voluntary activity with another adult person that involves sexual contact, whether or not intercourse or orgasm occurs" (Lindau et al., 2014).

Sexuality has been and will be a part of the individual for the entire life. However, the way the individual express his or her sexuality will change depending on the age, state of health and stage of life.However, the Consideration of how patients view sexuality is important 
when rendering nursing care, which should begin at the point of history taking and assessment. Lack of sexual education for patients will lead to absence or less frequent sexual activity in both male and female patients (Jaarsma et al., 2010). Therefore, providing patients with sexual health information aids in improving patients' sexual interest and satisfaction through decreasing patients' and partner level of anxiety and fear from engaging in sexual activities and improve patients' sexual life (Byrne et al., 2014) Despite supporting evidence and increased attention to sexual health assessment over the years, it is still a challenge for many nurses to address sexual health in practices.

Furthermore, there are so many misconceptions concerning the Sexual Health Assessment in Clinical Practice. This could be due to culture; belief and others.Sexual health assessment in clinical practice is viewed as a private matter between partners by nurses and rarely discussed (Bispo et al., 2013). However, sexual health assessment is important to help patients resume their sexual life (Reesea et al., 2012). There is an increasing interest in providing holistic care that includes sexual health assessment (Kotronoulas et al., 2015). Amongst other healthcare professionals, nurses can play a significant role in conducting sexual health assessment (Saunamäki and Engström, 2014). Nurses have good relationships with patients due to their daily contact with patients that facilitate assessment of sexual health (Goossen et al., 2011). Moreover, sexual health assessment for patients is a part of the nurses' professional role (Vitrano et al., 2011). It has shown that nurses have shown little competence in sexual health assessment and therefore requires more practice by nurse clinicians (Vitrano et al, 2011).

Furthermore, According to the American Heart Association, fulfilling sexual activity is an important aspect of the quality of life (Levine et al, 2012). Patients' sexual health assessment is a nursing task but nurses still hesitate to conduct sexual health assessment in clinical practice due to challenges which includes: Patients' lack of readiness, knowledge deficit and lack of training programs for nurses (Doherty et al., 2011). Study has shown that nurses are knowledgeable in sexual health field, feel comfortable and responsible and show readiness to do sexual health assessment, but the vast majority of them did not address patients' sexual health as they feel unconfident and did not specify a time to do so (Saunamäki et al., 2010).

Finally, nurses' beliefs and attitudes toward sexual health may prevent them from assessing patients' sexual health. Most of the nurses who never assess patients' sexual health have a negative attitude toward sexual health discussion (Byrne et al., 2010). Some nurses have personal beliefs and values that assessment of sexual issues is not ethical (Mick, 2014). Therefore, understanding nurses' attitudes towards addressing patients' sexual health is an important consideration by encouraging nurses to attend to patients' sexual concerns within the routine nursing care.It was reported that patient's' age, culture, sexual orientation, comorbidity diseases, and limited time can contribute to difficulty to adequate sexual health assessment (Buttaro et al., 2014).

\section{Challenges of nursing sexual assessment}

\section{Nurses}

- Some nurses thought that sexual health assessment is a taboo subject and therefore it should not be discussed and it is not within their job description and it is someone else's responsibility (Saunamäki et al, 2014).

- Insufficient nurses' knowledge and viewing sexual health as a private matter between adult partners which should not be discussed by nurses (Özdemir and Akdemir, 2016).

- Lack of time to prepare and feeling uncomfortable to provide patients with sexual education (Jaarsma et al, 2010).

- Some nurses view assessment of patients' sexual health as at low priority (Quinn et al., 2011) or make them upset, embarrassed or anxious (Jaarsma et al, 2010).

- Lack of enough time due to workload (Byrne, Doherty, Murphy and McGee, 2013).

- Feeling that patients are not ready to receive sexual information

- Inadequate or lack of education and training (Byrne et al, 2010).

\section{Patient}

- Patients are embarrassed to discuss their sexual health needs with the nurses (Taylor et al, 2011).

- Some patients are annoyed when there is discussion on their health related issues (Taylor et al, 2011).

- Patients' age, culture, sexual orientation, comorbidity diseases, and limited time are challenges to sexual health assessment (Buttaro et al, 2014).

\section{Organization}

- Lack of guidelines/ policies on sexual assessment and management in our health institution, especially, in the specialized units like medical wards, surgical wards, paediatrics' ward.

- Sexual health assessment isnot a priority in some institution. Not until health facilities sees the importance of sexual health assessment patient's personality might not be respected.

- Academic nursing curricula ignore discussing of 
sexual health issues for nursing students. This could serve vital to increase nurses' knowledge (Billington, 2012).

\section{Values}

Culture, society, and religion are related to values of persons or community preventing assessment of sexual health with patients during their hospitalization (Saunamäki et al, 2010).

In some cultures, there is a common belief that sexual health is not a matter for older adults or those who with chronic conditions (Magnan et al., 2012). Open communications about sexual matters between different sexes is prohibited in some culture, then preventing nurses from sexual health assessment (Ayhan et al., 2010).

\section{Way forward}

- Sexual health assessment topics should be integrated in nursing curriculum during undergraduate studies in order to increase the nurses' knowledge from scratch.

- Train, Retrain sessions and workshops on sexual health assessment for nurses in clinical setting to improve sexual health competency.

- Nurses should be taught effective communication skills and build rapport, therapeutic relationship between nurse and patients to make nurses more able to translate their acquired sexual knowledge into practice.

- Brochures and leaflets that are developed by experts about sexual health should be provided by health facilities to improve patients' knowledge and encourage patients to express their sexual health concerns.

- Time should be created for effective assessment and teaching of patients' sexual health.

- Create, adopt and implement sexual health policies and procedures for sexual health assessment in clinical settings to facilitate nurses' roles in conducting this important nursing task.

- Partners should be invited during sexual health assessment and counseling. This could contribute to the reduction of anxiety exhibited by the patient or client.

- Sexual health and related concerns can be discussed publicly on the media in order to break the privacy of health related issues that can ruin life, especially sex education for teenagers.

- Nurses need to understand patients and partners' sexual health experiences and needs for sexual health information, nurses' knowledge, attitudes, beliefs, barriers and best teaching methods.

- Create an appropriate work environment through establishing specialized rooms in health institutions to help both nurses and patients to address sexual health related issues in a more comfortable manner.

\section{Nursing theory applied}

\section{Hildegard E. Peplau theory of interpersonal relations}

According to Peplau, nursing is therapeutic because it is a healing art, assisting an individual who is sick or in need of health care. Nursing can be viewed as an interpersonal process because it involves interaction between two or more individuals with a common goal. In nursing, this common goal provides the incentive for the therapeutic process in which the nurse and patient respect each other as individuals, both of them learning and growing as a result of the interaction. An individual learns when she or he selects stimuli in the environment and then reacts to these stimuli.

Peplau defines man as an organism that "strives in its own way to reduce tension generated by needs." The client is an individual with a felt need. Although Peplau does not directly address society/environment, she does encourage the nurse to consider the patient's culture and mores when the patient adjusts to hospital routine. Hildegard Peplau considers nursing to be a "significant, therapeutic, interpersonal process." She defines it as a "human relationship between an individual who is sick, or in need of health services, and a nurse specially educated to recognize and to respond to the need for help."

\section{Therapeutic nurse-client relationship}

A professional and planned relationship between client and nurse that focuses on the client' needs feelings, problems, and ideas. Nursing involve interaction between two or more individuals with a common goal. The attainment of this goal, or any goal, is achieved through a series of steps following a sequential pattern.Four Phases of the therapeutic nurse-patient relationship:

- The orientation phase is directed by the nurse and involves engaging the client in treatment, providing explanations and information, and answering questions.

- The identification phase begins when the client works interdependently with the nurse, expresses feelings, and begins to feel stronger.

- In the exploitation phase, the client makes full use of the services offered.

- In the resolution phase, the client no longer needs professional services and gives up dependent behavior. The relationship ends.

\section{Roles of the nurse in the therapeutic relationship}

The primary roles she identified are as follows:

- Stranger: offering the client the same acceptance and courtesy that the nurse would to any stranger. 
- $\quad$ Resource person: providing specific answers to questions within a larger context

- Teacher: helping the client to learn formally or informally.

- Leader: offering direction to the client or group

- Surrogate: serving as a substitute for another such as a parent or a sibling

- Counselor: promoting experiences leading to health for the client such as expression of feelings.

Peplau also believed that the nurse could take on many other roles, including consultant, tutor, safety agent, mediator, administrator, observer, and researcher. These were not defined in detail but were "left to the intelligence and imagination of the readers." (Peplau, 1952).

In relation to this write up, the nurses needs to develop an interpersonal relationship which involves good rapport with patients or clients in order for them to be able to open up. Discussion on sexuality is very sensitive an embarrassing for some people of culture to discuss. If not properly discussed, the patient's identity might not be known as will be difficult to treat or care for. Therefore, nurses should use all the techniques of communication proposed by Peplau to gain the patient's confidence. The Nurse performs her role of a teacher, leader, resource person, surrogate and counselor to gain the patient or client's confidence. This will in turn allow the patient or client to freely discuss his or her sexual related issue and not take offence.

\section{SUMMARY}

Sexuality is a part of who you are as a person. It is how you feel about your body, whether you feel masculine or feminine or somewhere in between, the way you dress, how you move, how you speak, the way you act and feel about other people, who you are attracted to and fall in love with, and so much more. The way the individual express his or her sexuality will change depending on the age, state of health and stage of life. It is very important for Nurses to have adequate knowledge of sexuality in order to educate patient or client to promote their good sexual health activities when ill or not.

\section{RECOMMENDATION}

- Sex education should be given freely in the social and religious gathering such as market places, schools or colleges, churches and mosques.

- Sexual health issues should be included in student nurses' academic curriculum and should be discussed well.

- Workshops and seminars should be organized for the nurses in their various health facilities and state level.

- Each facility should have a guiding policy on sexual health assessment.

- Patient or Client's right should be respected.

\section{CONCLUSION}

Everybody wants to be respected. For this respect to be given, individual's sexuality must be well understood by the other person, especially the nurses that care for patients in the ward so that the time the patients spends on ward is reduced.

\section{REFERENCES}

Ayhan H, lyigun E, Tastan S and Coskun H (2010). Turkish Version of the Reliability and Validity Study of the Sexual Attitudes and Belief Survey. Sexuality and Disability, 28, 287-296.

Billington T (2012). Issues of Patient Sexuality in Nurse Education. British J. Nurs., 21, 1109.

Bispo GS, Lima Lopes J, Barros AL (2013). Cardiovascular Changes Resulting from Sexual Activity and Sexual Dysfunction after Myocardial Infarction: Integrative Review. Journal of Clinical Nursing, 22, 3522-3531.

Buttaro TM, Koeniger-Donohue R, Hawkins J (2014). Feature Article: Sexuality and Quality of Life in Aging: Implications for Practice. The J. for Nurse Practitioners, 10, 480-485

Byrne M, Doherty S, Fridlund BG, Mårtensson J, Steinke EE, Jaarsma T, Devane D (2014). Sexual Counseling for Sexual Problems in Patients with Cardiovascular Disease . The Cochrane Library, No. 2, $1-12$.

Byrne M, Doherty S, McGee HM, Murphy AW (2010). General Practitioner Views about Discussing Sexual Issues with Patients with Coronary Heart Disease: A National Survey in Ireland. BMC Family Practice, 11, 40.

Byrne M, Doherty S, Murphy AW, McGee HM, Jaarsma T (2013). The Charms Study: Cardiac Patients' Experiences of Sexual Problems Following Cardiac Rehabilitation. European J. Cardiovascular Nursing, 12, 558-566.

Doherty S, Byrne M, Murphy A, McGee H (2011): Cardiac Rehabilitation Staff Views about Discussing Sexual Issues with Coronary Heart Disease Patients: A National Survey in Ireland. European J. Cardiovascular Nursing, 10, 101- 107.

Goossens E, Norekvål TM, Faerch J, Hody L, Olsen SS, Darmer MR, Moons P (2011). Sexual Counselling of Cardiac Patients in Europe: Culture Matters. International Journal of Clinical Practice, 65, 10921099

Jaarsma TS, De Geest $S$, Mårtensson J, Moons $P$, Smithh K, Thompson D (2010). Sexual Counselling of Cardiac Patients: Nurses' Perception of Practice, Responsibility and Confidence. European J. Cardiovascular Nursing, 9, 24- 29.

Kotronoulas G, Papadopoulou C, Patiraki E (2016). Nurses' Knowledge, Attitudes and Practices Regarding Provision of Sexual Health Care in Patients with Cancer: Critical Review of the Evidence. Supportive Care in Cancer, 17, 479-501.

Levine GN, Steinke EE, Bakaeen FG, Bozkurt B, Cheitlin MD, Conti JB, Stewart WJ (2012): Sexual Activity and Cardiovascular Disease a Scientific Statement from the American Heart Association. Circulation, 125, 1058-1072.

Lindau ST, Schumm LP, Laumann EO, Levinson WO, 'Muircheartaigh CA, Waite LJ (2014). A Study of Sexuality and Health among Older Adults in the United States. New England J. Med, 357, 762-774.

Magnan ME, Reynolds KE, Galvin E (2012). Barriers to Addressing Patient Sexuality in Nursing Practice. MEDSURG Nursing, 14, 282289.

Mick J (2014). Sexuality Assessment: 10 Strategies for Improvement. Clinical J. Oncology Nursing, 11, 671-675.

Özdemir L, Akdemir N (2016). Nurses' Knowledge and Practice 
020 Educ. Res.

Involving Patients' Resuming Sexual Activity Following Myocardial Infarction: Implications for Training. Australian J. Advanced Nursing, 26, 42-52.

Quinn C, Happell B, Browne G (2011): Talking or Avoiding? Mental Health Nurses' Views about Discussing Sexual Health with Consumers. International J. Mental Health Nursing, 20, 21-28.

Reesea JP, Shelbyb RA, Taylorc KL (2012). Sexual Quality of Life in Patients Undergoing Coronary Artery Bypass Graft Surgery. Psychology and Health, 27, 721-736.

Saunamäki N, Engström M (2014). Registered Nurses' Reflections on Discussing Sexuality with Patients: Responsibilities, Doubts and Fears. Journal of Clinical Nursing, 23, 531-540.

Saunamäki N, Andersson M, Engström M (2010). Discussing Sexuality with Patients: Nurses' Attitudes and Beliefs.Journal of Advanced Nursing, 66, 1308- 1316

Taylor A, Gosney MA (2011). Sexuality in Older Age: Essential Considerations for Health Care Professionals. Age Ageing, 40, 538543.
Vitrano V, Catania V, Mercadante S (2011). Sexuality in Patients with Advanced Cancer: A Prospective Study in a Population Admitted to an Acute Pain Relief and Palliative Care Unit. American J. Hospice and Palliative Med., 28, 198-202.

Vitrano V, Catania V, Mercadante S (2011). Sexuality in Patients with Advanced Cancer: A Prospective Study in a Population Admitted to an Acute Pain Relief and Palliative Care Unit. American J. Hospice and Palliative Med., 28, 198-202.

WHO (2012). Defining Sexual Health Report of a Technical Consultation on Sexual Health 28-31 Jan. 2012, Geneva. Sexual Health Document Series, World Health Organization, Geneva.

Zeng YC, Liu X, Loke AY (2012). Addressing Sexuality Issues of Women with Gynaecological Cancer: Chinese Nurses' Attitudes and Practice. J. Advanced Nursing, 68, 280-292. 